\title{
Measurement of $\mathcal{B}(t \rightarrow W b) / \mathcal{B}(t \rightarrow W q)$ at the Collider Detector at Fermilab
}

D. Acosta, ${ }^{16}$ J. Adelman, ${ }^{12}$ T. Affolder,${ }^{9}$ T. Akimoto, ${ }^{54}$ M.G. Albrow,${ }^{15}$ D. Ambrose, ${ }^{15}$ S. Amerio,${ }^{42}$ D. Amidei,,${ }^{33}$ A. Anastassov,${ }^{50}$ K. Anikeev ${ }^{15}$ A. Annovi,${ }^{44} \mathrm{~J}$. Antos, ${ }^{1}$ M. Aoki,${ }^{54}$ G. Apollinari,${ }^{15}$ T. Arisawa, ${ }^{56} \mathrm{~J}-\mathrm{F}$. Arguin ${ }^{32}$ A. Artikov, ${ }^{13}$ W. Ashmanskas, ${ }^{15}$ A. Attal,${ }^{7}$ F. Azfar, ${ }^{41}$ P. Azzi-Bacchetta, ${ }^{42}$ N. Bacchetta,${ }^{42}$ H. Bachacou, ${ }^{28}$ W. Badgett, ${ }^{15}$ A. Barbaro-Galtieri, ${ }^{28}$ G.J. Barker,${ }^{25}$ V.E. Barnes,${ }^{46}$ B.A. Barnett, ${ }^{24}$ S. Baroiant,${ }^{6}$ G. Bauer, ${ }^{31}$ F. Bedeschi, ${ }^{44}$ S. Behari, ${ }^{24}$ S. Belforte,${ }^{53}$ G. Bellettini, ${ }^{44}$ J. Bellinger ${ }^{58}$ A. Belloni, ${ }^{31}$ E. Ben-Haim,${ }^{15}$ D. Benjamin, ${ }^{14}$ A. Beretvas, ${ }^{15}$ T. Berry ${ }^{29}$ A. Bhatti,${ }^{48}$ M. Binkley, ${ }^{15}$ D. Bisello, ${ }^{42}$ M. Bishai, ${ }^{15}$ R.E. Blair,${ }^{2}$ C. Blocker,${ }^{5}$

K. Bloom,${ }^{33}$ B. Blumenfeld, ${ }^{24}$ A. Bocci ${ }^{48}$ A. Bodek,${ }^{47}$ G. Bolla,,${ }^{46}$ A. Bolshov, ${ }^{31}$ D. Bortoletto, ${ }^{46}$ J. Boudreau, ${ }^{45}$ S. Bourov ${ }^{15}$ B. Brau,${ }^{9}$ C. Bromberg,${ }^{34}$ E. Brubaker, ${ }^{12}$ J. Budagov,${ }^{13}$ H.S. Budd,${ }^{47}$ K. Burkett,${ }^{15}$ G. Busetto, ${ }^{42}$ P. Bussey, ${ }^{19}$ K.L. Byrum, ${ }^{2}$ S. Cabrera,${ }^{14}$ M. Campanelli, ${ }^{18}$ M. Campbell, ${ }^{33}$ F. Canelli, ${ }^{7}$ A. Canepa,${ }^{46}$ M. Casarsa ${ }^{53}$ D. Carlsmith, ${ }^{58}$ R. Carosi, ${ }^{44}$ S. Carron,,${ }^{14}$ M. Cavalli-Sforza,${ }^{3}$ A. Castro, ${ }^{4}$ P. Catastini, ${ }^{44}$ D. Cauz,${ }^{53}$ A. Cerri, ${ }^{28}$ L. Cerrito, ${ }^{41}$ J. Chapman, ${ }^{33}$ Y.C. Chen,${ }^{1}$ M. Chertok,${ }^{6}$ G. Chiarelli, ${ }^{44}$ G. Chlachidze,${ }^{13}$ F. Chlebana,${ }^{15}$ I. Cho, ${ }^{27}$ K. Cho,${ }^{27}$ D. Chokheli, ${ }^{13}$ J.P. Chou, ${ }^{20}$ S. Chuang, ${ }^{58}$ K. Chung, ${ }^{11}$ W-H. Chung, ${ }^{58}$ Y.S. Chung, ${ }^{47}$ M. Cijliak, ${ }^{44}$ C.I. Ciobanu, ${ }^{23}$ M.A. Ciocci, ${ }^{44}$ A.G. Clark, ${ }^{18}$ D. Clark, ${ }^{5}$ M. Coca, ${ }^{14}$ A. Connolly, ${ }^{28}$ M. Convery ${ }^{48}$ J. Conway ${ }^{6}$ B. Cooper, ${ }^{30}$ K. Copic,${ }^{33}$ M. Cordelli, ${ }^{17}$ G. Cortiana, ${ }^{42}$ J. Cranshaw,${ }^{52}$ J. Cuevas, ${ }^{10}$ A. Cruz, ${ }^{16}$ R. Culbertson, ${ }^{15}$ C. Currat ${ }^{28}$ D. Cyr ${ }^{58}$ D. Dagenhart,${ }^{5}$ S. Da Ronco, ${ }^{42}$ S. D'Auria, ${ }^{19}$ P. de Barbaro, ${ }^{47}$ S. De Cecco, ${ }^{49}$ A. Deisher,${ }^{28}$ G. De Lentdecker, ${ }^{47}$ M. Dell'Orso, ${ }^{44}$ S. Demers, ${ }^{47}$ L. Demortier, ${ }^{48}$ M. Deninno, ${ }^{4}$ D. De Pedis,${ }^{49}$ P.F. Derwent,${ }^{15}$ C. Dionisi,${ }^{49}$ J.R. Dittmann, ${ }^{15}$ P. DiTuro, ${ }^{50}$ C. Dörr, ${ }^{25}$ A. Dominguez, ${ }^{28}$ S. Donati, ${ }^{44}$ M. Donega, ${ }^{18}$ J. Donini, ${ }^{42}$ M. D’Onofrio, ${ }^{18}$ T. Dorigo, ${ }^{42}$ K. Ebina,${ }^{56}$ J. Efron, ${ }^{38}$ J. Ehlers,${ }^{18}$ R. Erbacher, ${ }^{6}$ M. Erdmann, ${ }^{25}$ D. Errede,${ }^{23}$

S. Errede, ${ }^{23}$ R. Eusebi, ${ }^{47}$ H-C. Fang, ${ }^{28}$ S. Farrington, ${ }^{29}$ I. Fedorko, ${ }^{44}$ W.T. Fedorko, ${ }^{12}$ R.G. Feild,${ }^{59}$

M. Feindt, ${ }^{25}$ J.P. Fernandez ${ }^{46}$ R.D. Field, ${ }^{16}$ G. Flanagan, ${ }^{34}$ L.R. Flores-Castillo, ${ }^{45}$ A. Foland, ${ }^{20}$ S. Forrester, ${ }^{6}$ G.W. Foster, ${ }^{15}$ M. Franklin, ${ }^{20}$ J.C. Freeman, ${ }^{28}$ Y. Fujii, ${ }^{26}$ I. Furic,${ }^{12}$ A. Gajjar, ${ }^{29}$ M. Gallinaro, ${ }^{48}$ J. Galyardt,${ }^{11}$ M. Garcia-Sciveres ${ }^{28}$ A.F. Garfinkel,${ }^{46}$ C. Gay,${ }^{59}$ H. Gerberich, ${ }^{14}$ D.W. Gerdes,${ }^{33}$ E. Gerchtein, ${ }^{11}$ S. Giagu, ${ }^{49}$

P. Giannetti, ${ }^{44}$ A. Gibson, ${ }^{28}$ K. Gibson, ${ }^{11}$ C. Ginsburg, ${ }^{15}$ K. Giolo, ${ }^{46}$ M. Giordani, ${ }^{53}$ M. Giunta,${ }^{44}$

G. Giurgiu,${ }^{11}$ V. Glagolev, ${ }^{13}$ D. Glenzinski, ${ }^{15}$ M. Gold,${ }^{36}$ N. Goldschmidt, ${ }^{33}$ D. Goldstein,${ }^{7}$ J. Goldstein,${ }^{41}$ G. Gomez,${ }^{10}$ G. Gomez-Ceballos, ${ }^{10}$ M. Goncharov, ${ }^{51}$ O. González, ${ }^{46}$ I. Gorelov, ${ }^{36}$ A.T. Goshaw, ${ }^{14}$ Y. Gotra, ${ }^{45}$

K. Goulianos ${ }^{48}$ A. Gresele, ${ }^{42}$ M. Griffiths ${ }^{29}$ C. Grosso-Pilcher, ${ }^{12}$ U. Grundler, ${ }^{23}$ J. Guimaraes da Costa, ${ }^{20}$ C. Haber ${ }^{28}$ K. Hahn, ${ }^{43}$ S.R. Hahn, ${ }^{15}$ E. Halkiadakis, ${ }^{47}$ A. Hamilton, ${ }^{32}$ B-Y. Han ${ }^{47}$ R. Handler, ${ }^{58}$ F. Happacher, ${ }^{17}$ K. Hara, ${ }^{54}$ M. Hare, ${ }^{55}$ R.F. Harr, ${ }^{57}$ R.M. Harris,${ }^{15}$ F. Hartmann, ${ }^{25}$ K. Hatakeyama, ${ }^{48}$ J. Hauser, ${ }^{7}$ C. Hays, ${ }^{14}$

H. Hayward, ${ }^{29}$ B. Heinemann, ${ }^{29}$ J. Heinrich,${ }^{43}$ M. Hennecke, ${ }^{25}$ M. Herndon,,${ }^{24}$ C. Hill,,${ }^{9}$ D. Hirschbuehl,${ }^{25}$

A. Hocker ${ }^{15}$ K.D. Hoffman,${ }^{12}$ A. Holloway, ${ }^{20}$ S. Hou, ${ }^{1}$ M.A. Houlden,${ }^{29}$ B.T. Huffman,${ }^{41}$ Y. Huang, ${ }^{14}$ R.E. Hughes ${ }^{38}$ J. Huston, ${ }^{34}$ K. Ikado,${ }^{56}$ J. Incandela, ${ }^{9}$ G. Introzzi,${ }^{44}$ M. Iori,${ }^{49}$ Y. Ishizawa,${ }^{54}$ C. Issever, ${ }^{9}$ A. Ivanov ${ }^{6}$ Y. Iwata, ${ }^{22}$ B. Iyutin, ${ }^{31}$ E. James, ${ }^{15}$ D. Jang, ${ }^{50}$ B. Jayatilaka,${ }^{33}$ D. Jeans, ${ }^{49}$ H. Jensen, ${ }^{15}$ E.J. Jeon, ${ }^{27}$ M. Jones, ${ }^{46}$ K.K. Joo, ${ }^{27}$ S.Y. Jun, ${ }^{11}$ T. Junk, ${ }^{23}$ T. Kamon, ${ }^{51}$ J. Kang, ${ }^{33}$ M. Karagoz Unel,${ }^{37}$ P.E. Karchin, ${ }^{57}$ Y. Kato ${ }^{40}$ Y. Kemp,${ }^{25}$ R. Kephart,${ }^{15}$ U. Kerzel, ${ }^{25}$ V. Khotilovich, ${ }^{51}$ B. Kilminster, ${ }^{38}$ D.H. Kim, ${ }^{27}$ H.S. Kim, ${ }^{23}$ J.E. Kim, ${ }^{27}$ M.J. Kim, ${ }^{11}$ M.S. Kim,${ }^{27}$ S.B. Kim,${ }^{27}$ S.H. Kim,${ }^{54}$ Y.K. Kim, ${ }^{12}$ M. Kirby, ${ }^{14}$ L. Kirsch, ${ }^{5}$ S. Klimenko, ${ }^{16}$ M. Klute, ${ }^{31}$ B. Knuteson, ${ }^{31}$ B.R. Ko,${ }^{14}$ H. Kobayashi, ${ }^{54}$ D.J. Kong,${ }^{27}$ K. Kondo, ${ }^{56}$ J. Konigsberg, ${ }^{16}$ K. Kordas,${ }^{32}$ A. Korn, ${ }^{31}$ A. Korytov, ${ }^{16}$ A.V. Kotwal, ${ }^{14}$ A. Kovalev, ${ }^{43}$ J. Kraus, ${ }^{23}$ I. Kravchenko, ${ }^{31}$ A. Kreymer,${ }^{15}$ J. Kroll, ${ }^{43}$ M. Kruse,${ }^{14}$ V. Krutelyov, ${ }^{51}$ S.E. Kuhlmann, ${ }^{2}$ S. Kwang, ${ }^{12}$ A.T. Laasanen, ${ }^{46}$ S. Lai, ${ }^{32}$ S. Lami, ${ }^{44}$ S. Lammel,${ }^{15}$ M. Lancaster,${ }^{30}$ R. Lander,${ }^{6}$ K. Lannon, ${ }^{38}$ A. Lath, ${ }^{50}$ G. Latino, ${ }^{44}$ I. Lazzizzera, ${ }^{42}$ C. Lecci, ${ }^{25}$ T. LeCompte ${ }^{2}$

J. Lee, ${ }^{27}$ J. Lee, ${ }^{47}$ S.W. Lee ${ }^{51}$ R. Lefèvre,${ }^{3}$ N. Leonardo, ${ }^{31}$ S. Leone, ${ }^{44}$ S. Levy, ${ }^{12}$ J.D. Lewis,${ }^{15}$ K. Li,${ }^{59}$ C. Lin, ${ }^{59}$ C.S. Lin, ${ }^{15}$ M. Lindgren, ${ }^{15}$ E. Lipeles,${ }^{8}$ T.M. Liss, ${ }^{23}$ A. Lister, ${ }^{18}$ D.O. Litvintsev, ${ }^{15}$ T. Liu, ${ }^{15}$ Y. Liu, ${ }^{18}$ N.S. Lockyer, ${ }^{43}$ A. Loginov ${ }^{35}$ M. Loreti, ${ }^{42}$ P. Loverre,,${ }^{49}$ R-S. Lu, ${ }^{1}$ D. Lucchesi, ${ }^{42}$ P. Lujan, ${ }^{28}$ P. Lukens,${ }^{15}$ G. Lungu, ${ }^{16}$ L. Lyons,${ }^{41}$ J. Lys, ${ }^{28}$ R. Lysak, ${ }^{1}$ E. Lytken, ${ }^{46}$ D. MacQueen, ${ }^{32}$ R. Madrak,${ }^{15}$ K. Maeshima, ${ }^{15}$ P. Maksimovic, ${ }^{24}$ G. Manca, ${ }^{29}$ Margaroli,${ }^{4}$ R. Marginean, ${ }^{15}$ C. Marino, ${ }^{23}$ A. Martin, ${ }^{59}$ M. Martin, ${ }^{24}$ V. Martin, ${ }^{37}$ M. Martínez, ${ }^{3}$ T. Maruyama, ${ }^{54}$ H. Matsunaga, ${ }^{54}$ M. Mattson, ${ }^{57}$ P. Mazzanti, ${ }^{4}$ K.S. McFarland, ${ }^{47}$ D. McGivern, ${ }^{30}$ P.M. McIntyre, ${ }^{51}$ P. McNamara, ${ }^{50}$ McNulty, ${ }^{29}$ A. Mehta,${ }^{29}$ S. Menzemer,${ }^{31}$ A. Menzione,${ }^{44}$ P. Merkel, ${ }^{46}$ C. Mesropian, ${ }^{48}$ A. Messina, ${ }^{49}$ T. Miao,${ }^{15}$ N. Miladinovic,${ }^{5}$ J. Miles ${ }^{31}$ L. Miller, ${ }^{20}$ R. Miller,${ }^{34}$ J.S. Miller,${ }^{33}$ C. Mills, ${ }^{9}$ R. Miquel, ${ }^{28}$ S. Miscetti, ${ }^{17}$ G. Mitselmakher ${ }^{16}$ A. Miyamoto,,${ }^{26}$ N. Moggi, ${ }^{4}$ B. Mohr, ${ }^{7}$ R. Moore,${ }^{15}$ M. Morello, ${ }^{44}$ P.A. Movilla Fernandez ${ }^{28}$ J. Muelmenstaedt, ${ }^{28}$ A. Mukherjee, ${ }^{15}$ M. Mulhearn, ${ }^{31}$ T. Muller ${ }^{25}$ R. Mumford,${ }^{24}$ A. Munar ${ }^{43}$ P. Murat,${ }^{15}$ J. Nachtman, ${ }^{15}$ S. Nahn, ${ }^{59}$ I. Nakano, ${ }^{39}$ A. Napier,${ }^{55}$ R. Napora,${ }^{24}$ 
D. Naumov ${ }^{36}$ V. Necula, ${ }^{16}$ T. Nelson, ${ }^{15}$ C. Neu, ${ }^{43}$ M.S. Neubauer, ${ }^{8}$ J. Nielsen,${ }^{28}$ T. Nigmanov, ${ }^{45}$ L. Nodulman, ${ }^{2}$ O. Norniella, ${ }^{3}$ T. Ogawa, ${ }^{56}$ S.H. Oh, ${ }^{14}$ Y.D. Oh,${ }^{27}$ T. Ohsugi, ${ }^{22}$ T. Okusawa, ${ }^{40}$ R. Oldeman,${ }^{29}$ R. Orava, ${ }^{21}$

W. Orejudos,${ }^{28}$ K. Osterberg, ${ }^{21}$ C. Pagliarone,${ }^{44}$ E. Palencia, ${ }^{10}$ R. Paoletti,${ }^{44}$ V. Papadimitriou, ${ }^{15}$

A.A. Paramonov, ${ }^{12}$ S. Pashapour,${ }^{32}$ J. Patrick,${ }^{15}$ G. Pauletta,${ }^{53}$ M. Paulini,,${ }^{11}$ C. Paus,${ }^{31}$ D. Pellett,${ }^{6}$ A. Penzo,${ }^{53}$

T.J. Phillips, ${ }^{14}$ G. Piacentino, ${ }^{44}$ J. Piedra, ${ }^{10}$ K.T. Pitts,${ }^{23}$ C. Plager,${ }^{7}$ L. Pondrom,${ }^{58}$ G. Pope, ${ }^{45}$ X. Portell, ${ }^{3}$

O. Poukhov, ${ }^{13}$ N. Pounder,${ }^{41}$ F. Prakoshyn,${ }^{13}$ A. Pronko, ${ }^{16}$ J. Proudfoot,${ }^{2}$ F. Ptohos, ${ }^{17}$ G. Punzi, ${ }^{44}$

J. Rademacker, ${ }^{41}$ M.A. Rahaman, ${ }^{45}$ A. Rakitine,${ }^{31}$ S. Rappoccio ${ }^{20}$ F. Ratnikov,${ }^{50}$ H. Ray, ${ }^{33}$ B. Reisert,${ }^{15}$

V. Rekovic ${ }^{36}$ P. Renton, ${ }^{41}$ M. Rescigno, ${ }^{49}$ F. Rimondi, ${ }^{4}$ K. Rinnert,${ }^{25}$ L. Ristori, ${ }^{44}$ W.J. Robertson, ${ }^{14}$ A. Robson, ${ }^{19}$

T. Rodrigo, ${ }^{10}$ S. Rolli, ${ }^{55}$ R. Roser,${ }^{15}$ R. Rossin, ${ }^{16}$ C. Rott, ${ }^{46}$ J. Russ,${ }^{11}$ V. Rusu, ${ }^{12}$ A. Ruiz,${ }^{10}$ D. Ryan, ${ }^{55}$

H. Saarikko, ${ }^{21}$ S. Sabik,,${ }^{32}$ A. Safonov ${ }^{6}$ R. St. Denis,${ }^{19}$ W.K. Sakumoto,${ }^{47}$ G. Salamanna, ${ }^{49}$ D. Saltzberg, ${ }^{7}$

C. Sanchez,${ }^{3}$ L. Santi, ${ }^{53}$ S. Sarkar, ${ }^{49}$ K. Sato, ${ }^{54}$ P. Savard, ${ }^{32}$ A. Savoy-Navarro, ${ }^{15}$ P. Schlabach, ${ }^{15}$ E.E. Schmidt,${ }^{15}$

M.P. Schmidt, ${ }^{59}$ M. Schmitt, ${ }^{37}$ T. Schwarz, ${ }^{33}$ L. Scodellaro, ${ }^{10}$ A.L. Scott, ${ }^{9}$ A. Scribano, ${ }^{44}$ F. Scuri, ${ }^{44}$ A. Sedov, ${ }^{46}$

S. Seidel,${ }^{36}$ Y. Seiya,${ }^{40}$ A. Semenov ${ }^{13}$ F. Semeria,${ }^{4}$ L. Sexton-Kennedy, ${ }^{15}$ I. Sfiligoi, ${ }^{17}$ M.D. Shapiro, ${ }^{28}$ T. Shears,${ }^{29}$

P.F. Shepard, ${ }^{45}$ D. Sherman, ${ }^{20}$ M. Shimojima, ${ }^{54}$ M. Shochet, ${ }^{12}$ Y. Shon, ${ }^{58}$ I. Shreyber ${ }^{35}$ A. Sidoti, ${ }^{44}$ A. Sill, ${ }^{52}$

P. Sinervo, ${ }^{32}$ A. Sisakyan, ${ }^{13}$ J. Sjolin, ${ }^{41}$ A. Skiba, ${ }^{25}$ A.J. Slaughter, ${ }^{15}$ K. Sliwa, ${ }^{55}$ D. Smirnov, ${ }^{36}$ J.R. Smith, ${ }^{6}$

F.D. Snider, ${ }^{15}$ R. Snihur, ${ }^{32}$ M. Soderberg, ${ }^{33}$ A. Soha,${ }^{6}$ S.V. Somalwar, ${ }^{50}$ J. Spalding, ${ }^{15}$ M. Spezziga,${ }^{52}$ F. Spinella, ${ }^{44}$

P. Squillacioti, ${ }^{44}$ H. Stadie, ${ }^{25}$ M. Stanitzki, ${ }^{59}$ B. Stelzer, ${ }^{32}$ O. Stelzer-Chilton, ${ }^{32}$ D. Stentz, ${ }^{37}$ J. Strologas, ${ }^{36}$

D. Stuart,${ }^{9}$ J. S. Suh, ${ }^{27}$ A. Sukhanov, ${ }^{16}$ K. Sumorok,${ }^{31}$ H. Sun,,${ }^{55}$ T. Suzuki,${ }^{54}$ A. Taffard,${ }^{23}$ R. Tafirout,${ }^{32}$

H. Takano, ${ }^{54}$ R. Takashima,${ }^{39}$ Y. Takeuchi,,${ }^{54}$ K. Takikawa,${ }^{54}$ M. Tanaka,${ }^{2}$ R. Tanaka, ${ }^{39}$ N. Tanimoto,${ }^{39}$

M. Tecchio, ${ }^{33}$ P.K. Teng, ${ }^{1}$ K. Terashi, ${ }^{48}$ R.J. Tesarek,,${ }^{15}$ S. Tether,${ }^{31}$ J. Thom,${ }^{15}$ A.S. Thompson, ${ }^{19}$ E. Thomson, ${ }^{43}$

P. Tipton, ${ }^{47}$ V. Tiwari, ${ }^{11}$ S. Tkaczyk,${ }^{15}$ D. Toback, ${ }^{51}$ K. Tollefson,${ }^{34}$ T. Tomura,${ }^{54}$ D. Tonelli, ${ }^{44}$ M. Tönnesmann, ${ }^{34}$

S. Torre ${ }^{44}$ D. Torretta, ${ }^{15}$ S. Tourneur,${ }^{15} \mathrm{~W}$. Trischuk, ${ }^{32} \mathrm{R}$. Tsuchiya, ${ }^{56} \mathrm{~S}$. Tsuno,${ }^{39} \mathrm{D}$. Tsybychev,${ }^{16} \mathrm{~N}$. Turini ${ }^{44}$

F. Ukegawa, ${ }^{54}$ T. Unverhau, ${ }^{19}$ S. Uozumi ${ }^{54}$ D. Usynin, ${ }^{43}$ L. Vacavant ${ }^{28}$ A. Vaiciulis,${ }^{47}$ A. Varganov ${ }^{33}$

S. Vejcik III, ${ }^{15}$ G. Velev,${ }^{15}$ V. Veszpremi,${ }^{46}$ G. Veramendi,${ }^{23}$ T. Vickey, ${ }^{23}$ R. Vidal,${ }^{15}$ I. Vila, ${ }^{10}$ R. Vilar, ${ }^{10}$ I. Vollrath, ${ }^{32}$ I. Volobouev, ${ }^{28}$ M. von der Mey,${ }^{7}$ P. Wagner, ${ }^{51}$ R.G. Wagner, ${ }^{2}$ R.L. Wagner,${ }^{15}$ W. Wagner, ${ }^{25}$ R. Wallny ${ }^{7}$ T. Walter, ${ }^{25}$ Z. Wan, ${ }^{50}$ M.J. Wang, ${ }^{1}$ S.M. Wang, ${ }^{16}$ A. Warburton, ${ }^{32}$ B. Ward,${ }^{19}$ S. Waschke, ${ }^{19}$ D. Waters ${ }^{30}$ T. Watts,${ }^{50}$ M. Weber ${ }^{28}$ W.C. Wester III, ${ }^{15}$ B. Whitehouse, ${ }^{55}$ D. Whiteson, ${ }^{43}$ A.B. Wicklund, ${ }^{2}$ E. Wicklund, ${ }^{15}$ H.H. Williams, ${ }^{43}$ P. Wilson, ${ }^{15}$ B.L. Winer,${ }^{38}$ P. Wittich,${ }^{43}$ S. Wolbers,${ }^{15}$ C. Wolfe,${ }^{12}$ M. Wolter, ${ }^{55}$

M. Worcester, ${ }^{7}$ S. Worm, ${ }^{50}$ T. Wright,${ }^{33}$ X. Wu ${ }^{18}$ F. Würthwein, ${ }^{8}$ A. Wyatt,${ }^{30}$ A. Yagil,,${ }^{15}$ T. Yamashita,${ }^{39}$

K. Yamamoto, ${ }^{40}$ J. Yamaoka,${ }^{50}$ C. Yang, ${ }^{59}$ U.K. Yang, ${ }^{12}$ W. Yao, ${ }^{28}$ G.P. Yeh, ${ }^{15}$ J. Yoh, ${ }^{15}$ K. Yorita, ${ }^{56}$ T. Yoshida ${ }^{40}$

I. Yu, ${ }^{27}$ S. Yu, ${ }^{43}$ J.C. Yun, ${ }^{15}$ L. Zanello, ${ }^{49}$ A. Zanetti, ${ }^{53}$ I. Zaw,${ }^{20}$ F. Zetti, ${ }^{44}$ J. Zhou, ${ }^{50}$ and S. Zucchelli ${ }^{4}$

(CDF Collaboration)

${ }^{1}$ Institute of Physics, Academia Sinica, Taipei, Taiwan 11529, Republic of China

${ }^{2}$ Argonne National Laboratory, Argonne, Illinois 60439

${ }^{3}$ Institut de Fisica d'Altes Energies, Universitat Autonoma de Barcelona, E-08193, Bellaterra (Barcelona), Spain

${ }^{4}$ Istituto Nazionale di Fisica Nucleare, University of Bologna, I-40127 Bologna, Italy

${ }^{5}$ Brandeis University, Waltham, Massachusetts 02254

${ }^{6}$ University of California, Davis, Davis, California 95616

${ }^{7}$ University of California, Los Angeles, Los Angeles, California 90024

${ }^{8}$ University of California, San Diego, La Jolla, California 92093

${ }^{9}$ University of California, Santa Barbara, Santa Barbara, California 93106

${ }^{10}$ Instituto de Fisica de Cantabria, CSIC-University of Cantabria, 39005 Santander, Spain

${ }^{11}$ Carnegie Mellon University, Pittsburgh, PA 15213

${ }^{12}$ Enrico Fermi Institute, University of Chicago, Chicago, Illinois 60637

${ }^{13}$ Joint Institute for Nuclear Research, RU-141980 Dubna, Russia

${ }^{14}$ Duke University, Durham, North Carolina 27708

${ }^{15}$ Fermi National Accelerator Laboratory, Batavia, Illinois 60510

${ }^{16}$ University of Florida, Gainesville, Florida 32611

${ }^{17}$ Laboratori Nazionali di Frascati, Istituto Nazionale di Fisica Nucleare, I-00044 Frascati, Italy

${ }^{18}$ University of Geneva, CH-1211 Geneva 4, Switzerland

${ }^{19}$ Glasgow University, Glasgow G12 8QQ, United Kingdom

${ }^{20}$ Harvard University, Cambridge, Massachusetts 02138

${ }^{21}$ Division of High Energy Physics, Department of Physics,

University of Helsinki and Helsinki Institute of Physics, FIN-00014, Helsinki, Finland

${ }^{22}$ Hiroshima University, Higashi-Hiroshima 724, Japan

${ }^{23}$ University of Illinois, Urbana, Illinois 61801 


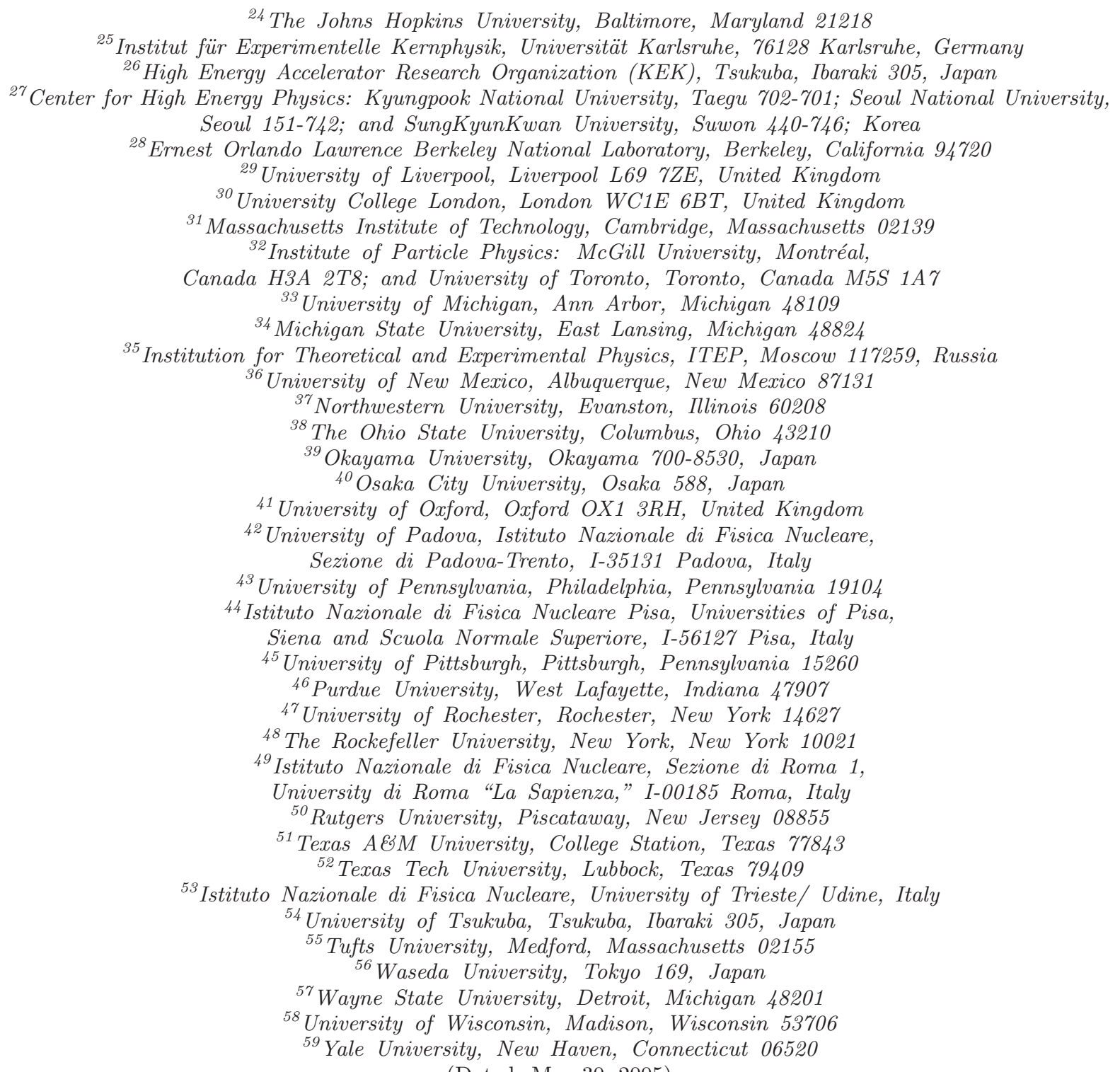

(Dated: May 30, 2005)

\begin{abstract}
We present a measurement of the ratio of top-quark branching fractions $R=\mathcal{B}(t \rightarrow W b) / \mathcal{B}(t \rightarrow$ $W q$ ) using lepton-plus-jets and dilepton data sets with integrated luminosity of $\sim 162 \mathrm{pb}^{-1}$ collected with the Collider Detector at Fermilab during Run II of the Tevatron. The measurement is derived from the relative numbers of $t \bar{t}$ events with different multiplicity of identified secondary vertices. We set a lower limit of $R>0.61$ at $95 \%$ confidence level.
\end{abstract}

PACS numbers: $14.65 . \mathrm{Ha}, 12.15 . \mathrm{Hh}$

The top quark as described by the Standard Model (SM) is expected to decay to a $W$ boson and a bottom quark at least $99.8 \%$ of the time at $90 \%$ confidence level (CL) 1]. The Cabbibo-Kobayashi-Maskawa (CKM) quark-mixing matrix [2, 3] element $\left|V_{t b}\right|$ is expected to be very close to unity from the assumption of a unitary, three-generation matrix and the measured small values of $\left|V_{u b}\right|$ and $\left|V_{c b}\right|$ 1]. A measurement of the ratio of topquark branching fractions $R=\mathcal{B}(t \rightarrow W b) / \mathcal{B}(t \rightarrow W q)$, where $q$ can be a $b, s$ or a $d$ quark, significantly less than unity would contradict our current theoretical assump- tions, implying either non-SM top decay, a non-SM background to top-pair production, or a fourth generation of quarks. A previous measurement has set a lower limit of $R>0.56$ at $95 \%$ CL 4 . In this Letter we present a measurement of $R$ using $t \bar{t}$ events collected at the Collider Detector at Fermilab (CDF) during Run II of the Tevatron, a proton-antiproton collider with center of mass energy of $\sqrt{s}=1.96 \mathrm{TeV}$. The integrated luminosity of the data sample used in this analysis is $\sim 162 \mathrm{pb}^{-1}$.

At the Tevatron, top quarks are observed in $t \bar{t}$ pairs. The lifetime of top is too short for hadronization to oc- 
cur, and the SM strongly favors an essentially immediate decay of each quark to a real $W$ boson and weak-isospin $-1 / 2$ quark; if $R=1$, this is always a $b$ quark. To maintain high detection and trigger efficiencies and low background levels, we only consider $t \bar{t}$ final states in which at least one $W$ has decayed leptonically. Events in which one $W$ decays leptonically are called "lepton-plus-jets" $(\mathrm{L}+\mathrm{J})$ events, and events with two leptonic decays are called "dilepton" (DIL) events. Values of $R$ are determined separately for each of these sets of events, and are combined in the end to set a lower limit on $R$. The greater statistical power comes from the L+J sample.

The measurement requires both the counting of $b$ quark jets and the determination of the $t \bar{t}$ content as a function of the $b$-quark multiplicity. We identify ("tag") $b$-quark jets by identifying displaced secondary vertices using the SECVTX algorithm [5]. $R$ is extracted from the relative rates of events with zero, one, and two tags; any two rates determine $R$ uniquely, while all three rates jointly overdetermine $R$. A novel feature of this measurement is the determination of the 0 -tag $\mathrm{L}+\mathrm{J}$ event rate using event kinematics and an artificial neural net (ANN) technique. As $R$ depends only on relative rates, this measurement is independent of any assumptions of the overall $t \bar{t}$ cross section. However, our measurement of $R$ does depend critically on the knowledge of the efficiency to identify $b$ jets, $\epsilon_{b}$. To extract $R$ we use a value of $\epsilon_{b}$ estimated with a $t \bar{t}$ Monte Carlo (MC) sample in which tagging efficiencies have been tuned to match jet data [5].

The CDF detector for Run II [6] consists of a chargedparticle tracking system in a magnetic field of $1.4 \mathrm{~T}$, segmented electromagnetic and hadronic calorimeters and muon detectors. A silicon microstrip detector provides tracking over the radial range 1.5 to $28 \mathrm{~cm}$, and is essential for the detection of displaced secondary vertices. The fiducial region of the silicon detector covers the pseudorapidity range $|\eta|<2$, while the central tracking system and muon chambers provide coverage for $|\eta|<1$ [7]. A three-level trigger system is used to select events with electron (muon) candidates with $E_{T}\left(p_{T}\right)>18 \mathrm{GeV}(18$ $\mathrm{GeV} / c)$, which form the data set for this analysis.

$\mathrm{L}+\mathrm{J}$ events consist of one isolated high- $p_{T}$ lepton $(e$ or $\mu)$, large missing transverse energy $\left(\notin_{T}\right)$ due to the undetected neutrino, and four hadronic jets. Two of these jets arise from the hadronic decay of the other $W$, and the other two arise from the top-daughter quarks $q$. The $\mathrm{L}+\mathrm{J}$ selection requirements are described in detail elsewhere [5]. Briefly, we require the presence of an isolated lepton which has transverse momentum greater than $20 \mathrm{GeV} / c$, that $\mathbb{E}_{T}$ is at least $20 \mathrm{GeV}$, and that there are a minimum of four jets, clustered with a cone-based algorithm having cone dimension $\Delta R=\sqrt{(\Delta \phi)^{2}+(\Delta \eta)^{2}}=$ 0.4 , within $|\eta|<2$ and with corrected transverse energy 5] greater than $15 \mathrm{GeV}$. These requirements select 107 events.
TABLE I: Summary of observed number of events with $i$ tags in the $\mathrm{L}+\mathrm{J}$ and DIL samples, with estimates of nominal $t \bar{t}$ event-tagging efficiencies, background levels and expected event yields. The $\mathrm{L}+\mathrm{J} 0$-tag background is measured with an ANN. The efficiency estimates and the 1-tag and 2-tag L+J background estimates are given for $R=1$. Equations 1 and 2 are used for the calculation of the expected total number of events $N_{i}^{\exp }$. The statistical and systematic uncertainties have been combined.

\begin{tabular}{cccc}
\hline \hline Lepton + Jets $(\mathbf{L}+\mathbf{J})$ & 0-tag & 1-tag & 2-tag \\
\hline Efficiency $\left(\epsilon_{i}(R=1)\right)$ & $0.45 \pm 0.03$ & $0.43 \pm 0.02$ & $0.12 \pm 0.02$ \\
Background $\left(N_{i}^{\text {bkg }}\right)$ & $62.4 \pm 9.0$ & $4.2 \pm 0.7$ & $0.2 \pm 0.1$ \\
Total expected $\left(N_{i}^{\text {exp }}\right)$ & $80.4 \pm 5.2$ & $21.5 \pm 4.1$ & $5.0 \pm 1.4$ \\
Observed $\left(N_{i}^{\text {obs }}\right)$ & 79 & 23 & 5 \\
\hline Dileptons $(\mathbf{D I L})$ & $\mathbf{0 - t a g}$ & $\mathbf{1 - t a g}$ & 2-tag \\
\hline Efficiency $\left(\epsilon_{i}(R=1)\right)$ & $0.47 \pm 0.03$ & $0.43 \pm 0.02$ & $0.10 \pm 0.02$ \\
Background $\left(N_{i}^{\text {bkg }}\right)$ & $2.0 \pm 0.6$ & $0.2 \pm 0.1$ & $<0.01$ \\
Total expected $\left(N_{i}^{\text {exp }}\right)$ & $6.1 \pm 0.4$ & $4.0 \pm 0.2$ & $0.9 \pm 0.2$ \\
Observed $\left(N_{i}^{\text {obs }}\right)$ & 5 & 4 & 2 \\
\hline \hline
\end{tabular}

DIL events consist of two charged leptons (ee, $\mu \mu$ or $e \mu$ ), large $\mathbb{E}_{T}$ due to the undetected neutrinos, and two jets from the top-daughter quarks $q$. The DIL selection requirements are described in detail elsewhere [8]. Compared to the $\mathrm{L}+\mathrm{J}$ selection, we demand an additional lepton, but only a minimum of two energy-corrected [8] jets, with the same requirements as before. These requirements select 11 events.

Both event samples are subdivided on the basis of the number of identified $b$ jets in the event. The number of events in each subsample with $i$ tagged jets are given in Table【 The 2-tag subsample is defined to include events with $\geq 2$ tags; in this data sample we observe no events with more than two tagged jets.

In the $\mathrm{L}+\mathrm{J}$ sample, the dominant background is $W$ production in association with jets from QCD processes (" $W+$ jets" events). In the 1-tag and 2-tag subsamples we make an a priori estimate of the backgrounds with a collection of data-driven and simulation techniques that are described in detail elsewhere 5]. The backgrounds in these subsamples include $W$ production in association with heavy-flavor jets $(W b \bar{b}, W c \bar{c}, W c), W$ production in association with light-flavor jets that are incorrectly identified as $b$ jets ("mistags"), QCD events containing fake or real leptons and/or incorrectly-measured $\mathbb{E}_{T}$, dibosons $(W W, W Z$ ) and single-top quark production. The background estimate requires a small correction for $R \neq 1$. The background estimate for $R=1$ in these subsamples is given in Table [ The uncertainties on the estimate are dominated by uncertainties in the fraction of $W+$ jets events that include heavy flavor and on the normalization of the QCD background rate.

By construction, the a priori method cannot predict the background level in the 0 -tag $\mathrm{L}+\mathrm{J}$ sample, where the production rate of $W+$ jets dominates the $t \bar{t}$ production; instead we make use of event kinematics. The artificial 


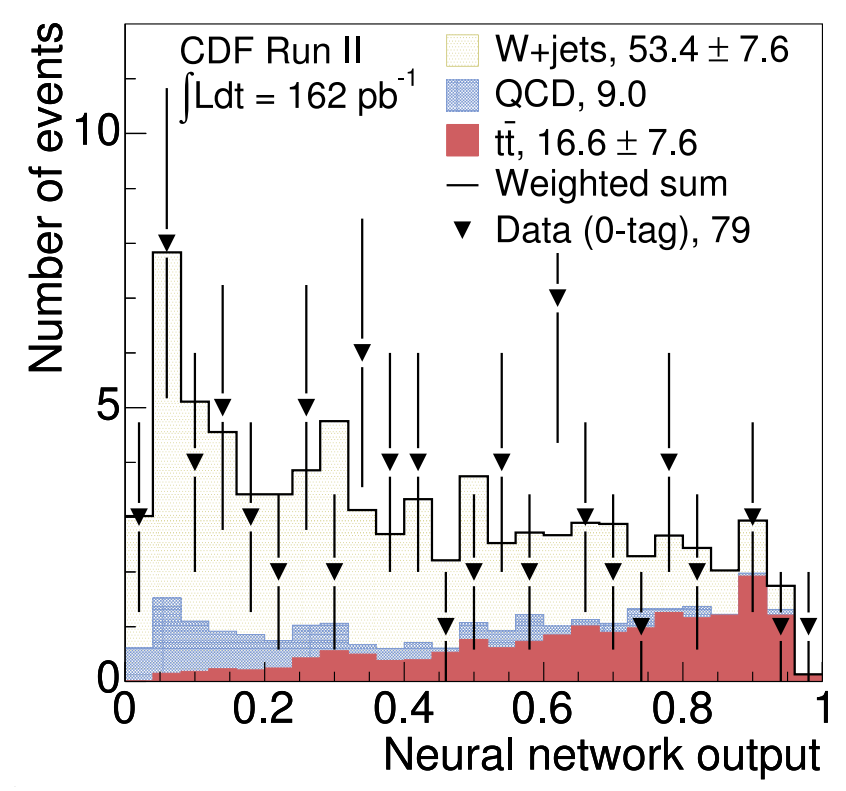

FIG. 1: Fit of the ANN output in the 0-tag L+J data set (triangles) with a sum of 3 components: $W+$ jets (upper), QCD (middle), and $t \bar{t}$ (lower). The QCD normalization is independently estimated and not varied in the fit; its shape is determined from non-isolated lepton data.

neural net is trained with the $t \bar{t}$ signal (HERWIG [9]) and $W+$ jets background (HERWIG+ALPGEN [10]) events simulated with a detailed detector description based on GEANT [1]. There is an additional QCD background which is modeled using data with non-isolated leptons. We find optimal signal to background discrimination with an ANN structure of nine input variables, one intermediate layer with ten nodes, and one output unit. The variables used are the transverse energies of the four leading jets, the minimum di-jet mass, the di-jet transverse mass with value closest to the mass of the $W$, the scalar sum of the transverse energies of all leptons and jets, the total longitudinal momentum divided by the total transverse momentum, and the event aplanarity.

The ANN output ranges from zero for background-like events to one for signal-like events. We perform a binned maximum likelihood fit of the ANN output distribution for the $t \bar{t}$ fraction in the 0 -tag subsample. The fraction of events from QCD backgrounds is fixed to $11.4 \%$ in this fit. These events are characterized by the non-isolation of the lepton and small $\mathscr{E}_{T}$, and the fixed rate is based on comparing to control regions with either low $\mathbb{E}_{T}$ or poor isolation [5]. The resulting measurement of background rates in the 0-tag $\mathrm{L}+\mathrm{J}$ subsample is given in Table】 The fit of the distribution of ANN outputs for this subsample is shown in Figure 1

Systematic uncertainties in the ANN-determined backgrounds are dominated by our understanding of the jet energy scale, the renormalization and factorization scale, and the shape of the QCD template and are strongly anticorrelated between the $t \bar{t}$ and $W+$ jets measurements. Our ANN-measured $t \bar{t}$ content in the $\mathrm{L}+\mathrm{J}$ sample without any tagging requirement is consistent with that found in our earlier measurement of the $t \bar{t}$ production cross section [12]. The procedure is repeated in the 1-tag and 2 -tag samples, yielding background rates of $5.8 \pm 5.2$ and $0.1_{-0.1}^{+1.0}$ respectively, consistent with the a priori estimates shown in Table \. As the a priori estimates have smaller uncertainties in the 1-tag and 2-tag subsamples, the ANN-determined background level is used only for the L+J 0-tag subsample.

The main backgrounds in the DIL sample are DrellYan production including lepton pairs from the $Z$ resonance, dibosons, and $W+$ jets events with fake leptons. The total background level of $2.2 \pm 0.6$ events in the DIL sample has been estimated elsewhere [8]. The Drell-Yan rate in $e e$ and $\mu \mu$ events is estimated using simulated data normalized to the observed rate of $Z$ events in the data. Other electroweak backgrounds are estimated from MC simulations. The fake-lepton background is estimated by multiplying each jet in $W$ plus three or more jet events by a lepton fake rate, measured in complementary jet samples.

Most of the jets in the DIL background events arise from generic QCD radiation. To determine the background distribution across the $i$-tag subsamples, we apply a parameterization of the probability to tag a generic QCD jet, derived from jet-triggered data samples, to the jets in the DIL sample, correcting for the enriched $t \bar{t}$ content of the sample. The resulting estimates are given in Table \ the background in the 2-tag subsample is negligible.

The $t \bar{t}$ event-tagging efficiency $\epsilon_{i}$, defined as the probability to observe $i$ tags in a $t \bar{t}$ event, depends on the fiducial acceptances for jets that can potentially be tagged, and the efficiencies to tag those jets. Those efficiencies in turn depend on the species of the underlying quark in the jet. The efficiency $\epsilon_{i}$ depends strongly on $R$, as $R \neq 1$ implies fewer $b$ jets available for tagging, and more lightquark jets available instead. We use the jet acceptances and tagging efficiencies to parameterize $\epsilon_{i}(R)$. These quantities are estimated with a sample of simulated $t \bar{t}$ events from the PYTHIA 13] generator and CDF detector simulation, and their uncertainties are dominated by our understanding of the control samples of jet data used to calibrate tagging efficiencies in the simulation. The leading determiner of $\epsilon_{i}$ is the efficiency to tag a $b$ jet from the decay $t \rightarrow W b ; \epsilon_{b}=0.44 \pm 0.04$ for $b$ jets falling within the fiducial acceptance and having at least two tracks with silicon information. The $\epsilon_{i}$ values also have small contributions from the efficiencies to tag jets from $W \rightarrow c s$ hadronic decays and from additional QCD radiation in $t \bar{t}$ events. The nominal values of $\epsilon_{i}$ for $R=1$ are given in Table

The expected event yield in each of the three tagged 
subsets of each of the $\mathrm{L}+\mathrm{J}$ and DIL samples is

$$
N_{i}^{\exp }=N_{\text {inc }}^{t \bar{t}} \cdot \epsilon_{i}(R)+N_{i}^{\mathrm{bkg}},
$$

where $N_{i}^{\mathrm{bkg}}$ is the number of background events in the $i$-tag subsample and $N_{\text {inc }}^{t \bar{t}}$ is an estimate of the inclusive number of $t \bar{t}$ events in the sample, determined by

$$
N_{\text {inc }}^{t \bar{t}}=\sum_{i}\left(N_{i}^{\mathrm{obs}}-N_{i}^{\mathrm{bkg}}\right),
$$

where $N_{i}^{\text {obs }}$ is the observed number of events in each subsample. In this construction, the measured value of $R$ is independent of any assumption of the overall rate of $t \bar{t}$ production, and is thus sensitive only to the relative numbers of $t \bar{t}$ events with $i$ tags.

The full likelihood is a product of independent likelihoods for the $\mathrm{L}+\mathrm{J}$ and DIL samples. Each likelihood is a product of Poisson functions comparing $N_{i}^{\text {obs }}$ to $N_{i}^{\exp }$ for each value of $i$, multiplied by Gaussian functions which incorporate systematic uncertainties in the event-tagging efficiencies and backgrounds, taking into account the correlations across the different subsamples. These include correlations in the event-tagging efficiencies through the single-jet tagging efficiencies; in the common methodology of the a priori estimates in the tagged $\mathrm{L}+\mathrm{J}$ samples; and in the overall normalization of the DIL backgrounds. There are a total of five free parameters in the likelihood to account for these systematic uncertainties.

The resulting likelihood as a function of $R$ is shown in Figure 2 along with the negative logarithm of the likelihood. We find a central value of $R=1.12_{-0.19}^{+0.21}$ (stat) $)_{-0.13}^{+0.17}$ (syst). The dominant systematic uncertainties arise from the uncertainty on the background measurement in the 0 -tag $\mathrm{L}+\mathrm{J}$ sample $\left({ }_{-0.11}^{+0.14}\right)$ and from the overall normalization of the tagging efficiencies $\left(\begin{array}{l}+0.09 \\ -0.06\end{array}\right)$. Taken separately, the two final states of $t \bar{t}$ give consistent results for $R$; the $\mathrm{L}+\mathrm{J}$ sample alone yields $R=1.02_{-0.20-0.13}^{+0.23+0.21}$, and the DIL sample alone yields $R=1.41_{-0.40-0.13}^{+0.46+0.17}$. These $R$ results are consistent with the SM expectations.

The ratio $R$ can only take on physical values between zero and unity. We use the Feldman-Cousins prescription [14] to set a lower limit on $R$. We generate ensembles of pseudo-experiments for different input values of $R\left(R_{\text {true }}\right)$, and vary the input quantities of the analysis, e.g. the background estimates, taking correlations into account. Using the likelihood-ratio ordering principle, we find the acceptance intervals as shown in Figure 2 With our measured value of $R$, we find that $R>0.61$ at the 95\% CL.

Our lower limit on $R$ is the strongest limit on this top-quark branching ratio to date. Within the SM, $R=\frac{\left|V_{t b}\right|^{2}}{\left|V_{t b}\right|^{2}+\left|V_{t s}\right|^{2}+\left|V_{t d}\right|^{2}}$, up to phase-space factors. Assuming unitarity of the CKM matrix, the denominator is unity, and we estimate $\left|V_{t b}\right|>0.78$ at $95 \%$ CL. All of our measurements of $R$ are consistent with the SM expectations.

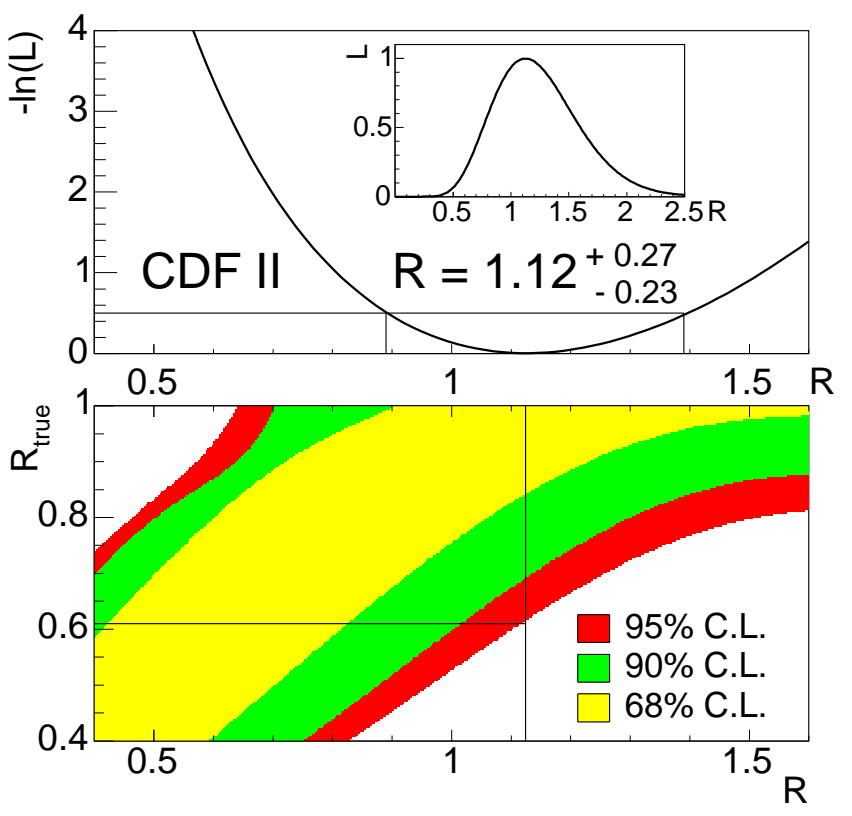

FIG. 2: The upper plot shows the likelihood as a function of $R$ (inset) and its negative logarithm. The intersections of the horizontal line $\ln (L)=-0.5$ with the likelihood define the statistical $1 \sigma$ errors on $R$. The lower plot shows $95 \%$ (outer), $90 \%$ (central), and 68\% (inner) CL bands for $R_{\text {true }}$ as a function of $R$. Our measurement of $R=1.12$ (vertical line) implies $R>0.61$ at the $95 \%$ CL (horizontal line).

We thank the Fermilab staff and the technical staffs of the participating institutions for their vital contributions. This work was supported by the U.S. Department of Energy and National Science Foundation; the Italian Istituto Nazionale di Fisica Nucleare; the Ministry of Education, Culture, Sports, Science and Technology of Japan; the Natural Sciences and Engineering Research Council of Canada; the National Science Council of the Republic of China; the Swiss National Science Foundation; the A.P. Sloan Foundation; the Bundesministerium für Bildung und Forschung, Germany; the Korean Science and Engineering Foundation and the Korean Research Foundation; the Particle Physics and Astronomy Research Council and the Royal Society, UK; the Russian Foundation for Basic Research; the Comision Interministerial de Ciencia y Tecnología, Spain; in part by the European Community's Human Potential Programme under contract HPRN-CT-2002-00292; and the Academy of Finland.

[1] S. Eidelman et al. (Particle Data Group), Phys. Lett. B 592, 1 (2004).

[2] N. Cabibbo, Phys. Rev. Lett. 10, 531 (1963).

[3] M. Kobayashi and T. Maskawa, Prog. Theor. Phys. 49, 652 (1973). 
[4] T. Affolder et al. (CDF Collaboration), Phys. Rev. Lett. 86, 3233 (2001).

[5] D. Acosta et al. (CDF Collaboration), Phys. Rev. D 71, 052003 (2005).

[6] D. Acosta et al. (CDF Collaboration), Tech. Rep. FERMILAB-PUB-04/440-E (2004).

[7] In the CDF geometry, $\theta$ is the polar angle with respect to the proton beam axis, and $\phi$ is the azimuthal angle. The pseudo-rapidity is $\eta \equiv-\ln (\tan (\theta / 2))$. The transverse momentum, $p_{T}$, is the component of the momentum projected onto the plane perpendicular to the beam axis. The transverse energy $E_{T}$ of a shower or calorimeter tower is $E \sin \theta$, where $E$ is the energy deposited.

[8] D. Acosta et al. (CDF Collaboration), Phys. Rev. Lett. 93, 142001 (2004), we restrict the $193 \mathrm{pb}^{-1}$ data set used here to runs in which the silicon detector was included.

[9] G. Corcella et al., JHEP 01, 010 (2001), arXiv:hep$\mathrm{ph} / 0011363$.

[10] M. L. Mangano et al., JHEP 07, 001 (2003), arXiv:hep$\mathrm{ph} / 0206293$.

[11] R. Brun and F. Carminati, CERN Programming Library Long Writeup W5013 (1993).

[12] D. Acosta et al. (CDF Collaboration), submitted to Phys. Rev. D (2005), arXiv:hep-ex/0504053.

[13] T. Sjöstrand et al., Computer Physics Commun. 135, 238 (2001), arXiv:hep-ph/0108264.

[14] G. Feldman and R. Cousins, Phys. Rev. D 57, 3873 (1998). 